\section{A incorporação de novas tecnologias nos serviços de saúde: o desafio da análise dos fatores em jogo}

\author{
Adoption of new technologies by health services: \\ the challenge of analyzing relevant factors
}

\begin{abstract}
The exponential increase in the incorporation of health technologies has been considered a key factor in increased expenditures by the health sector. Such decisions involve multiple levels and stakeholders. Decentralization has multiplied the decision-making levels, with numerous difficult choices and limited resources. The interrelationship between stakeholders is complex, in creative systems with multiple determinants and confounders. The current review discusses the interaction between the factors influencing the decisions to incorporate technologies by health services, and proposes a structure for their analysis. The application and intensity of these factors in decision-making and the incorporation of products and programs by health services shapes the installed capacity of local and regional networks and modifies the health system. Empirical observation of decision-making and technology incorporation in Brazilian health services poses an important challenge. The structured recognition and measurement of these variables can assist proactive planning of health services.
\end{abstract}

Biomedical Technology; Diffusion of Innovation; Health Planning; Health Services
Evelinda Trindade 1

\section{Introdução}

A dinâmica exponencial de incorporação tecnológica no setor saúde tem sido considerada como uma das razões para o crescimento dos gastos do setor. A descentralização adicionou níveis de decisão aos múltiplos atores envolvidos na incorporação de tecnologias para a saúde, e todos se encontram com difíceis escolhas múltiplas e recursos restritos.

As tecnologias para a saúde podem ser consideradas a aplicação prática de conhecimentos, por isto, incluem máquinas, procedimentos clínicos e cirúrgicos, remédios, programas e sistemas para prover cuidados à saúde. A legislação sanitária do Brasil padronizou o vocabulário de serviços e produtos para a saúde, denominando estes produtos como medicamentos, equipamentos, artigos e produtos para diagnóstico in vitro.

Essas tecnologias, em pequenos ou grandes grupos e nas mais variadas combinações, fazem parte dos programas de assistência à saúde. Nas últimas décadas, a assistência à saúde vem se tornando progressivamente mais dependente dessa instrumentação. As rápidas mudanças nas tecnologias utilizadas para prover a assistência à saúde, apesar de trazer inegáveis benefícios à longevidade e qualidade de vida da população, também trouxeram novos desafios e problemáticas.

O grande desafio para o governo é melhorar a qualidade e a eficiência dos serviços de saúde. Inúmeras publicações permeiam a literatura 
científica e os documentos oficiais referindo-se à complexidade 1 de atingir essas metas diante dos constrangimentos orçamentários para o setor saúde.

O contexto que adiciona dificuldades nessas escolhas é que a trajetória de uma tecnologia não é um fenômeno isolado, seu impacto no sistema de saúde pode ser uma cascata que propicia outros desenvolvimentos tecnológicos. Na prática observada, a expansão das áreas de intervenção se relaciona tanto com a introdução das novas tecnologias como com novas indicações de tecnologias estabelecidas (até mesmo com tecnologias controvertidas).

A complexidade deste tema e das estratégias para abordar estas análises de decisão tem despertado muito interesse. A incorporação de tecnologias no setor saúde pode ser determinada por uma ampla gama de fatores, alguns determinados pela natureza da própria tecnologia ou do problema relevante, e outros pelas ações e interesses dos diversos grupos envolvidos nos processos decisórios.

Este ensaio visa a estudar, consolidar os conteúdos publicados sobre os fatores que influenciam a decisão de adoção de tecnologias para a saúde e propor uma estrutura de análise com o objetivo de subsidiar sua discussão nos estabelecimentos de saúde do Brasil.

\section{Método}

Revisão descrevendo a literatura científica disponível nas bases bibliográficas MEDLINE/PubMed (http://www.nlm.nih.gov), LILACS (http://www. bireme.br), EMBASE (acesso online via página da Internet da Biblioteca da Faculdade de Medicina da Universidade de São Paulo; http://www. biblioteca.fm.usp.br) e dos periódicos biomédicos disponíveis no Portal CAPES (http://www. periodicos.capes.gov.br), bem como naqueles com pleno texto em OVID (acesso online via Portal da Pesquisa; http://www.portaldapesquisa. com.br/fmusp), via pesquisa simplificada indexando "adoption of innovation" OR "knowledge utilization" OR "technology transfer" AND "technology/biomedical" no título, palavras-chave ou no texto do abstrato [All Fields], no período de 1960 a março de 2006. Foram inclusos os artigos com aporte teórico e descrição dos fatores relevantes.

\section{Resultado e discussão}

A incorporação de tecnologias no setor saúde pode ser determinada por uma ampla gama de fatores, alguns determinados pela natureza da própria tecnologia ou do problema relevante, e outros pelas ações e interesses dos diversos grupos envolvidos.

Este tema e estratégias têm despertado muito interesse e foram amplamente revisados na literatura sobre difusão de inovações 2 , também referidos sob a terminologia knowledge utilization, technology transfer ou bridging the research/ practice gap, confundindo-se, algumas vezes, com a pesquisa translational (movendo ciência básica para a aplicada). O foco principal destas teorias mais recentes está na dinâmica dos processos, substituindo os modelos anteriores de mudanças lineares, e nos processos de comunicação ativos nas organizações, em contraste com as descrições prévias de adoção mais passiva mediada por indivíduos (modelo baseado em Greer ${ }^{3,4,5}$, Kaluzny ${ }^{6}$, Rogers \& Shoemaker ${ }^{7}$ e Warner ${ }^{8}$, adaptado por Fendrick \& Schwartz ${ }^{9}$ ).

Nos modelos ativos, cinco etapas são discriminadas para abordar todo o espectro desse processo 2: (i) o conhecimento da existência da inovação; (ii) a persuasão, relativa à opinião favorável, ou não, formada na unidade de decisão; (iii) a decisão de adotar, ou rejeitar, a inovação; (iv) a implementação da inovação; e (v) a confirmação, que reforça a decisão de adoção feita ou a rejeita. Este modelo conceitual inclui a premissa na etapa (i) de que o desenvolvimento da inovação, estudos pré-clínicos e clínicos de fases II e III, já estejam completos ou próximos deste estágio, e, reconhece a intervenção de múltiplos fatores em cada uma destas etapas.

Durante as etapas (iv) de implementação e (v) de confirmação, no entanto, existe o reconhecimento da entrada de outros atores (os novos utilizadores da tecnologia) e idéias, fornecendo importante feed back e/ou identificando nova(s) necessidade(s). A figura do profissional médico ou técnico desdobra-se assim em dois papéis 10: o de utilizador primário e o de criador de demanda (favorecendo a adoção, ou não, e/ou a adaptação) de modificação ou novo desenvolvimento (criando outras demandas de desenvolvimento).

Esses e os demais fatores envolvidos podem ser facilitadores/promotores ou constituir barreiras que dificultam a entrada, adoção ou a difusão de novas tecnologias, ou ambos, segundo a perspectiva da observação ou o sistema gerencial para a adoção de tecnologias 4 .

Certamente os hospitais são as estruturas do sistema de saúde com a maior concentração de tecnologias, e as estratégias para facilitar ou restringir a entrada destas varia segundo os sistemas de gestão adotados. Greer 11 estudou hospitais e identificou três sistemas gerenciais para a adoção de tecnologias (i) abordagem de gerencia- 
mento fiscal, (ii) estratégico institucional $12 \mathrm{ou}$ (iii) individualista médico. Essas estratégias, sob a perspectiva dos sistemas, entretanto, não são categorias exclusivas e na prática se observa sua concomitância e interação 13 .

Os mesmos fatores são associados positivamente ou negativamente à difusão das inovações, colocando-as ou suprimindo-as da agenda. Estes têm sido agrupados no nível dos médicos, no nível dos pacientes ou no nível relacionado com o sistema de saúde (Figura 1).

A ocorrência concomitante e a interação entre esses vários níveis e fatores estão mediadas por jogos de poder e interesses, cujo entendimento vem evoluindo desde a perspectiva do determinismo tecnológico 14 (em que o objeto da análise se centrava no médico que utilizava a tecnologia como um instrumento de coerção social) até a nova teoria da tecnologia na prática 15 (na qual a tecnologia é considerada como mais um ator, pois muda configurações de alguns elementos técnicos e sociais, e, em alguns casos coordena aspectos clínicos e organizacionais do serviço de saúde). Enquanto a primeira dessas teorias alertava contra a hege- monia médica e a medicalização da sociedade, nesta última se analisa a escolha das tecnologias como uma relação dinâmica e recíproca entre interesses sociais, do complexo industrial da saúde e da rede de atores. Sob essa abordagem, estudos interdisciplinares vêm analisando como o agenciamento da tecnologia é constituído por outros que, por sua vez, se constituem em ações para terceiros 16. Ou seja, inclui o olhar sobre a tecnologia como um mediador central na construção e reprodução de novos panoramas, incluindo identidade de pacientes 17 , identidades profissionais 18 e a organização do trabalho em serviços de saúde 19. Assim, evidencia-se e caracteriza-se a política em ação, no local e em ampla escala, ao observar-se a adoção e a implementação de uma inovação, ou sua rejeição, no debate entre os interesses desses atores.

Salientando os interesses dos atores envolvidos nas decisões relativas às novas tecnologias, observa-se que:

1. Aos gestores públicos e privados interessa que o sistema de saúde seja o mais efetivo e eficiente, resolvendo os problemas do públicoalvo, distribuindo os serviços para melhorar a

Figura 1

Fatores que afetam a adoção de novas tecnologias.

\begin{tabular}{|c|c|c|}
\hline \multirow[t]{2}{*}{ Nível de pacientes } & $\begin{array}{l}\text { Fatores relacionados à aceitação e satisfação } \\
\text { do paciente }\end{array}$ & $\begin{array}{l}\text { Custo para o paciente } \\
\text { Tempo para a visita ou internação } \\
\text { Privacidade do paciente } \\
\text { Desconforto para o paciente } \\
\text { Confiança ou conforto com a tecnologia } \\
\text { Atitude, percepção positiva para o uso da tecnologia }\end{array}$ \\
\hline & \multicolumn{2}{|l|}{ - Qualidade da comunicação do médico ao paciente } \\
\hline \multirow[b]{2}{*}{ Nível de médicos } & \multicolumn{2}{|c|}{ - Potencial de litígio \& confidencialidade para o paciente } \\
\hline & Características do médico & $\begin{array}{l}\text { Lugar e tamanho da clínica } \\
\text { Idade do médico } \\
\text { Nível de treinamento } \\
\text { Conhecimento e atitude } \\
\text { Filiação acadêmica } \\
\text { Especialidade }\end{array}$ \\
\hline \multirow[t]{2}{*}{$\begin{array}{l}\text { Nível do sistema de } \\
\text { saúde }\end{array}$} & Características da tecnologia & $\begin{array}{l}\text { Confiabilidade } \\
\text { Eficiência e tempo } \\
\text { Facilidade no uso } \\
\text { Percepção do uso } \\
\text { Conhecimento e acesso à tecnologia } \\
\text { Custos e custo-efetividadde }\end{array}$ \\
\hline & Características do programa que utiliza a tecnologia & $\begin{array}{l}\text { Custos de financiamento e reembolso } \\
\text { Custo-efetividade } \\
\text { Eficiência dos serviços }\end{array}$ \\
\hline
\end{tabular}

Fonte: adaptado e traduzido com permissão de Rogers 2. 
qualidade da saúde e da vida da população com a maior abrangência possível e mantendo o orçamento dentro de limites razoáveis ou possíveis;

2. Os pesquisadores médicos, na academia, estão certamente interessados em avançar as fronteiras do conhecimento e adquirir prestígio na disciplina científica relevante;

3. As empresas desenvolvem novos produtos objetivando desenvolver novos mercados, ter mais lucros e assegurar a sua sobrevivência;

4. Diretamente inseridos nos serviços de saúde, os médicos assistentes devem visar, em princípio e sobretudo, a melhorar a qualidade de vida, prevenir, curar ou paliar os problemas de saúde dos seus pacientes, podendo, ou não, ter interesse em novas tecnologias, sobretudo nos casos em que já existam soluções estabelecidas e conhecidas como satisfatórias. Verifica-se, no entanto, que o acúmulo das tarefas e os custos elevados de cursos e congressos de educação permanente dificultam o acesso à atualização para uma grande proporção desses profissionais, deixando-os ex- postos apenas às informações unilaterais entregues pelos visitadores médicos das companhias; 5. Os pacientes buscam soluções para problemas vividos ou percebidos visando a melhorar seu estado de saúde e;

6. Os cidadãos que trabalham e pagam os impostos que financiam o governo, ou/e além disto pagam asseguradores, esperam que estes recursos sejam aplicados da maneira mais adequada possível para maiores e melhores benefícios à saúde própria, de sua família e da sociedade para a qual contribuiu.

Cada um desses grupos de atores (stakeholders) atua sobre decisões de incorporação de tecnologias em diferentes níveis e temporalidade, sendo que no nível do governo, em primeira instância, a tecnologia deve ter sido aprovada na análise da vigilância sanitária (Figura 2) e estar disponível para a comercialização.

Após, considerando que a tecnologia deve ser inserida em programas de saúde, esta deve ser aprovada na lista para ressarcimentos/reembolsos do sistema de saúde público ou su-

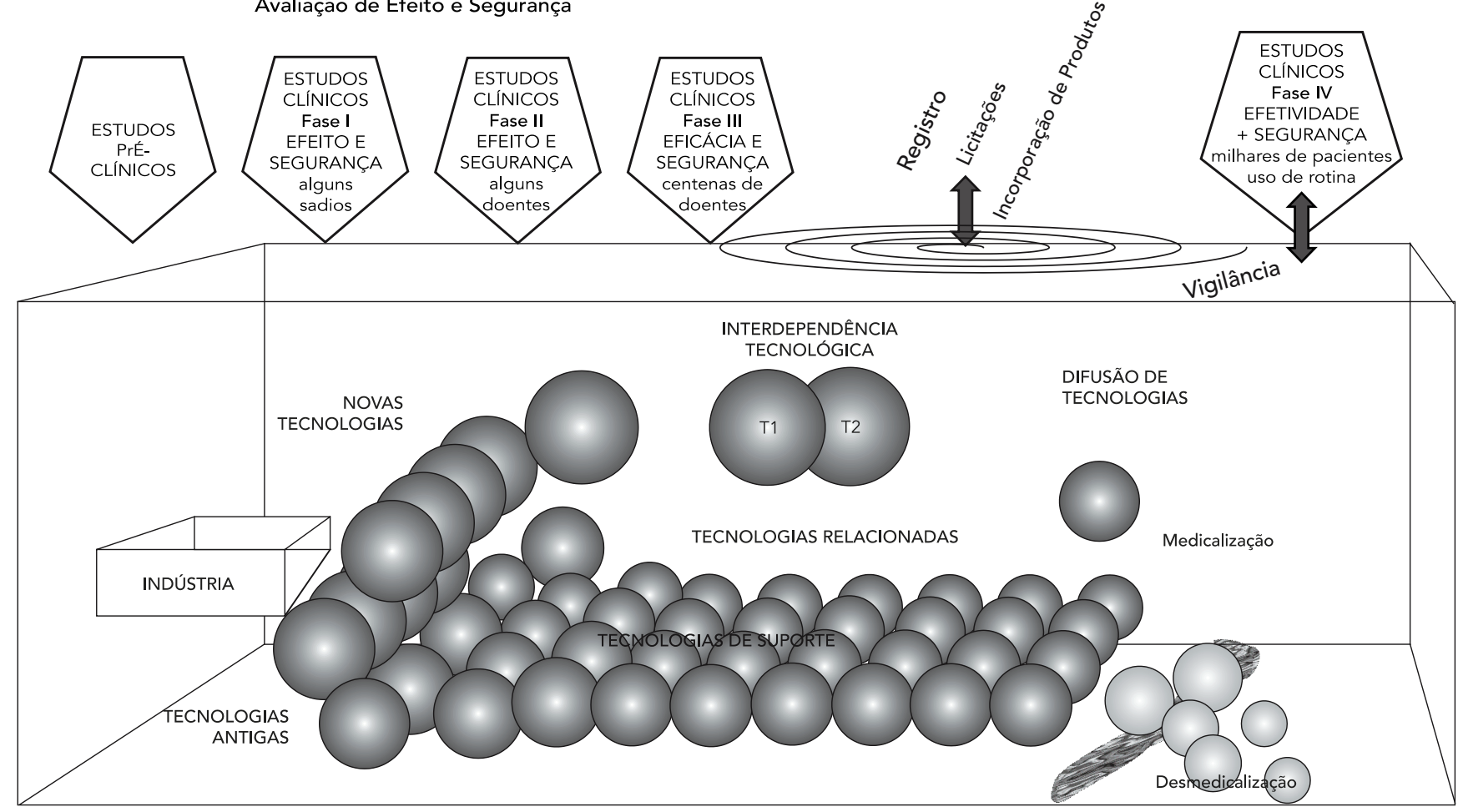


plementar. O rigor e a coordenação temporal 20 das avaliações conduzidas neste nível de decisão são determinantes para todo o desenvolvimento do sistema de saúde. Esta etapa de decisão tem impacto significativo nos custos para a saúde, bem como no acesso a outras novas tecnologias que poderiam ser mais apropriadas para as condições da população 21,22, propiciando, ou não, a implementação dos princípios de eqüidade e responsabilidade social.

Já estando autorizada a ser comercializada, e, sobretudo, com cobertura para ressarcimento, as tecnologias podem ser incorporadas nos estabelecimentos de saúde. No entanto, em um estudo destas decisões no nível de instituições, os atores colocaram em primeiro lugar de importância 23 as considerações clínicas e suas implicações, tanto em hospitais públicos como privados, e só em quinto lugar classificaram-se os requerimentos de aprovação formal das autoridades sanitárias. Isso denota lacunas, que merecem maiores reflexões, na formação dos médicos e administradores, pois ocorre mesmo nas instituições públicas. (Em 1990, 50 hospitais 24 canadenses ainda não possuíam sistemas estruturados para compras e relatavam que os processos eram políticos, informais ou com avaliação ad hoc. Em 2003, a situação parece ser similar em outros países 23 como também tem sido mencionado no Brasil 25).

No nível dos estabelecimentos de saúde, a idéia da necessidade de adoção ou influência para a incorporação raramente emerge isoladamente, de maneira verticalizada. Na maioria dos casos os determinantes estruturais da organização interagem de maneira complexa 26,27. O conhecimento que promove ou adota inovações não é o fato objetivo per si, e sim o conjunto de interações entre a liderança e as redes sociais que podem construir ou negociar o seu significado 28,29 , ou contestá-lo. A socialização do conhecimento nas redes interpessoais é que promove mudanças do significado 30 , levantando as barreiras à adoção da inovação. Mesmo nos contextos mais receptivos 31,32,33, com liderança muito forte ${ }^{34}$, o clima condutivo de tensão para mudança 35 deve ser criado, para que a experimentação e a aceitação do risco possam ocorrer. Sob esta perspectiva de aceitação do risco, cabe ressaltar que os estabelecimentos de saúde públicos possuem fontes de financiamento possivelmente mais flexíveis segundo a capacidade de negociação política de sua liderança, havendo, portanto, relativamente menor impacto. Por outro lado, os estabelecimentos de saúde universitários possuem duplo financiamento, originário da saúde e da educação, flexibilizando o risco de perdas por experimentação, tornando- se, em princípio, a estrutura mais propensa à receptividade e adoção de inovações.

Também no nível dos estabelecimentos de saúde, soma-se o fator considerado a assimetria de informação, questão assinalada por Arrow 36 desde 1963, demonstrando como o médico assistente influencia as decisões de incorporação e de uso das tecnologias em todos os níveis. Isso acontece durante a assistência, pois os pacientes não detêm a informação necessária para informar as escolhas, sendo compelidos a delegar ao médico a decisão sobre o tratamento. Cabe ao médico também decidir sobre quais serviços serão demandados e oferecidos, originando potencial conflito de interesses. A remuneração por honorários médicos pode, então, incentivar o excesso de oferta de serviços quando um terceiro agente é responsável pelo pagamento da maioria dos serviços que os médicos escolhem para seus pacientes. No lado do paciente, a demanda pode, então, fazer surgir uma inflação extra no crescimento da cobertura da atenção à saúde, risco moral 37 induzido ou voluntário, em sistemas em que os pacientes não arcam com o total dos custos marginais do excesso de utilização. Essa assimetria de informação também influencia as relações com os gestores do sistema de saúde e é determinante no papel do médico como promotor/introdutor de tecnologias no setor saúde.

O médico, por sua vez, sofre influências. Ele é observado pela indústria como o cliente 20,37 que compra e promove a venda dos produtos 39 . Do ponto de vista conceitual em gestão e marketing, os seguintes estereótipos de perfil de clientes têm caracterizado a literatura relacionada com a adoção de tecnologias:

1. Inovadores (tecnologistas, entusiastas): buscam inovações agressivamente; primeiros em tudo, seu endosso é crucial (market "seed" = "give" to gatekeepers);

2. Early Adopters (visionários, revolucionários): querem ser os primeiros, primeiro círculo de compradores, buscam vantagem competitiva (demandam modificações especiais);

3. Early Majority (pragmáticos): acreditam em evolução, não revolução; são práticos: o sistema deve trabalhar efetivamente, requerem referências estabelecidas e de confiança...;

4. Late Majority (conservadores): exigem padrões estabelecidos e menor preço;

5. Laggards = Skeptics (céticos): eternos críticos $\mathrm{e}$ só compram por necessidade específica.

Reconhecendo um contexto de interações complexas para a "compra e venda" de idéias inovadoras no setor saúde, este trabalho se tornou o clássico utilizado para a teoria de marketing.

Em 1962, Rogers 2 descreveu essas interações salientando a influência do contexto organiza- 
cional: no qual a receptividade às mudanças geram idéias criativas. $\mathrm{E}$ isso estimula a estruturação e trocas de conhecimento e influências com os demais sistemas na sociedade. Essa dinâmica favorece a adaptação à inovação e a emergência de outros desenvolvimentos, bem como a disseminação das experiências.

$\mathrm{Na}$ teoria de marketing esses conceitos foram introduzidos como uma função com distribuição normal. Esta curva foi então estudada e identificado o ponto crítico, denominado the chasm 40 , a ser superado para introduzir inovações no mercado.

Esses conceitos são aplicados extensivamente nos treinamentos aos promotores de companhias que visitam regularmente os médicos, os categorizam e cultivam um relacionamento estreito com técnicas aperfeiçoadas de marketing e adaptadas ao perfil observado.

Esta compreensão está também fundamentada na demonstração de Silverberg 41 de que o processo de difusão de inovações é ativo, pois estas não se difundem sem que haja o esforço de quem as adota. Os atributos e a proposta da própria tecnologia jogam, então, um papel determinante na sua adoção ${ }^{42}$. Seis atributos-padrão, centrais na teoria de marketing, se desenvolveram em uma base extensa de conhecimentos da sociologia médica:

1. O primeiro é a vantagem relativa percebida sobre a(s) tecnologia(s) existente(s), sine qua non para entusiasmar para a adoção 43,44 , embora este atributo por si só não garanta sua adoção 45 ; 2. O segundo é a compatibilidade ou a percepção de como esta nova tecnologia se encaixa convenientemente na(s) estrutura(s) de trabalho existente(s), procedimento(s), necessidade(s), padrões e valor(es) do(s) ator(es) 46,47,48,49,50;

3. O terceiro atributo descrito é a complexidade ou o grau de dificuldade envolvido em aprender a utilizar e implementar a nova tecnologia 51,52, sendo que a possível redução desta complexidade por meio de parcelamentos e adoção incremental das partes também está relatada como atributo facilitador 53,54 ;

4. O nível de facilidade de testar a nova tecnologia pelos potenciais utilizadores sem maiores investimentos de tempo e recursos foi o quarto atributo descrito 55,56;

5. O quinto seria a visibilidade ou possibilidade de observação dos resultados ao utilizar esta nova tecnologia $57 \mathrm{e}$;

6. O sexto se associa à possibilidade de adaptar, refinar, reinventar ou modificar para ajustar às suas próprias necessidades (ou ego).

Esses atributos da nova tecnologia proposta podem se superpor, aumentando em cadeia exponencial sua atratividade. Além disso, outras qualidades podem contribuir para aumentar esse efeito, tais como programas de treinamento adaptados e suporte técnico oferecido (customization e help desk) baseados no conceito de otimizar interações com os potenciais usuários 58 (p. 19): "o que pode ser fácil para alguns pode ser extremamente difícil para outros”. Assim, conceitualizar que a nova tecnologia tenha atributos pode, no entanto, obscurecer o papel central da percepção do indivíduo ou coletividade que decide sobre este investimento. Então, existe um processo contínuo inovação-difusão, observado nos estudos realizados e que se demonstrou dependente de adaptações e aperfeiçoamentos; onde existe maior experiência há maior desenvolvimento ao longo da trajetória tecnológica. Na perspectiva da teoria econômica 59 , este seria um fenômeno de retornos crescentes. O papel dos médicos que adotam a tecnologia em estágio precoce, early adopters, propicia a entrada, influencia o desenvolvimento, lhes trazendo, com isto, prestígio, vantagens e reconhecimento e, certas vezes, a inclusão da tecnologia na lista dos serviços cobertos 60 (lobby por publicações).

Nessa mesma linha de raciocínio, cabe ressaltar que o treinamento/educação dos profissionais em formação 61 tem considerável impacto na familiaridade, percepção da vantagem, ou não, desenvolvimento de competências e condicionamento da sua prática no futuro, influenciando a adoção, ou não ${ }^{62}$, e a difusão, ou não, da tecnologia. Essa rede social horizontal de inter-relações quase-familiar informal entre formandos e professores permanece a mais efetiva e duradoura maneira conhecida para disseminar influência e reconstruir significado 63,64. Além disso, face à rápida evolução dos avanços tecnológicos e das exigências de recredenciamento periódico, os programas de educação contínua passaram a influenciar a prática médica de maneira similar à formação de base. Treinamentos de pós-graduação passaram a desempenhar um papel importante na familiarização de profissionais com novas tecnologias para a saúde. Muitos desses cursos de atualização são ministrados nos congressos das especialidades médicas, que são realizados em paralelo a grandes feiras comerciais, onde os vários interesses são promovidos. Somam-se, então, fatores de status, pressão dos pares (homophily) 63,64,65, onde se incluem os líderes de opinião 66,67 , sobretudo aqueles que exercem papel preponderante por autoridade ou credibilidade (expert) 65,68 de maneira polimórfica 68,69 , ou seja, influentes em várias linhas de inovação. Caracterizados como campeões 70,71,72 que promovem ativamente a inovação ou como facilitadores 73 que criam coalizões dentro das organizações, que por sua vez aprimoram capa- 
cidades de expandir os horizontes 74 em matéria de inovações na organização. A maturidade profissional está, dessa forma, enfatizada como o fator mais importante de regulação e controle da prática médica, favorecendo, ou não 75 , mudanças tecnológicas.

Além do papel individual do médico, existem fenômenos coletivos e corporativos. Atualmente, existem redes de promoção de protocolos baseados em evidências científicas 76, guidelines, ou de consensos sobre a melhor prática 77 clínica ou cirúrgica. Embora muitas conferências de consenso sejam promovidas com base em interesses industriais, colocando na pauta temas específicos para fórum de validação com bons profissionais líderes; analisando e disseminando documentos sobre os riscos e efetividade de recursos para a saúde, estas Boas Práticas, best practices, influenciam na incorporação das tecnologias tanto no setor público como no privado. Internacionalmente, as agências de pesquisa e controle de qualidade de serviços de saúde lideram e também influenciam a "compra" e promovem a "venda" de produtos e serviços com selos distintivos por méritos por meio de avaliações favoráveis. Essas organizações disseminam amplamente as condutas estabelecidas, desejáveis, e em algumas ocasiões recomendando restrições à incorporação da nova tecnologia ou elencando requerimentos adicionais de conhecimentos.

Por esse mesmo mecanismo de promover o uso de best practices, no entanto, a sociedade civil organizada e organizações profissionais 78,79 podem, por vezes, sob interesses ou pressões diversas, ter um papel coletivo de pressão para a adoção de novas tecnologias ainda pouco estudadas, e também influenciam o ritmo de sua difusão. A mídia leiga ou interessada pode também ter um papel importante de disseminação das informações assim geradas, modificando percepções, pressionando as decisões e gerando demanda.

Isso constitui um elemento adicional de reflexão e diz respeito ao conceito da reflexividade do novo, elaborado por Giddens ${ }^{80}$, no qual diz que a modernidade é marcada pelo apetite pelo novo, não pelo novo por si só, mas pela suposição da reflexividade indiscriminada. Esta permeia e constrói certas percepções individuais ou coletivas, denominadas culturais, tais como "tudo que é novo é melhor" 81 (p. 7), e/ou percepções promovidas por publicidade. Em muitas ocasiões, ordens judiciais 82 podem ser acordadas para a provisão de novas tecnologias ao(s) paciente(s) que recebe $(\mathrm{m})$ ou solicita $(\mathrm{m})$ sua prescrição médica e recorre(m), ignorando evidências científicas, planejamento e até mesmo sem considerar as legislações dos sistemas na- cionais. Assim também, nos estabelecimentos de saúde, constitui-se a percepção cultural de status mais elevado ao possuir a última geração da(s) nova(s) tecnologia(s). E, quanto maior variação na sua distribuição geográfica, sobretudo as de alta complexidade, maior a competição entre os estabelecimentos de saúde, influenciando em mais aquisições e pressionando para aumentar a difusão. A percepção de status tem provocado amplo debate sobre este imperativo tecnológico 83,84, sob parâmetros morais, éticos, sociais ou econômicos e isto implica todos os níveis de decisão, coletivos ou individuais 85,86,87.

A desorganização e pouca transparência das informações epidemiológicas e sanitárias sobre a população e do planejamento para a assistência contribuem para este tipo de intercorrência. Esta falta da avaliação local explícita de necessidades em saúde, da sua sistematização e da sua disseminação também influenciam a difusão das tecnologias por meio de decisões desajustadas de adoção precoce de novas tecnologias.

As empresas fabricantes, importadoras ou distribuidoras mantêm estratégias agressivas de marketing/lobbies direto aos médicos e/ou ao público para a promoção de novas tecnologias 88,89. A disponibilização de informações, a estruturação de conteúdos quase-científicos, cursos e congressos patrocinados passam a transcender na rotina atribulada de um médico ocupado. Informações com vieses que podem ser transmitidas ao público também geram percepções de "milagre" que induzem demandas.

Estratégias de mercado têm influência considerável sobre a taxa de desenvolvimento e incorporação. A percepção das empresas sobre o interesse social de um desenvolvimento tecnológico, sobretudo para os maiores pagadores como o governo, também pode acelerar o ritmo de desenvolvimento e/ou aperfeiçoamento de produtos. Assegurando, ou não, a cobertura financeira de uma tecnologia, é o pagador que credencia a difusão da tecnologia. Por exemplo, o scanner Positron Emission Tomography (PET) da General Electric (GE), pronto desde 1990, era utilizado apenas em pesquisas. A GE não havia solicitado seu registro a Food and Drug Administration [FDA, Agência Americana de Controle de Alimentos e Medicamentos], pois o Center for Medical Services do U.S. Department of Health \& Human Services [HHS, Departamento de Saúde dos Estados Unidos], que gerencia os serviços públicos nos Estados Unidos, afirmava que não iria cobrir estes exames. Somente em 2000 houve a solicitação, sua aprovação para comercialização na FDA e sua incorporação pelo HHS (http:/ / www.fda.gov/cdrh/pdf/K001681.pdf, acessado em 08/Jan/2008). 
A estratégia das empresas em promover testes ou demonstrações de tecnologias, permite a sua promoção e identificação do programa onde a tecnologia poderia ser inserida. Esse mecanismo permite acesso e ensaio com a tecnologia inovadora, mas a probabilidade de vieses no conhecimento assim disseminado pode ser elevada, sobretudo em organizações com recursos reduzidos para avaliação de compras.

$\mathrm{O}$ relacionamento direto entre as empresas e pequenas instituições, com recursos reduzidos, também pode estimular estratégias pouco éticas para diminuir a concorrência. Uma interação maior pode facilitar o aporte de recursos técnicos ou outros quando há problemas na manutenção dos bens e produtos, e pode criar cumplicidade estratégica.

Considerando que o custo do investimento inicial em uma tecnologia pode ser derisório frente ao custo de insumos e materiais de programas que utilizam alguns equipamentos, doações 66 ou comodatos tem sido estratégias utilizadas para garantir parcelas de mercado. Isso ocorre, de maneira pró-ativa ou reativa, de parte das empresas, mas, sobretudo, nas áreas onde insumos similares também são compatíveis. Equipamentos laboratoriais, bombas de infusão e máquinas de hemodiálise são exemplos freqüentes de uso dessas estratégias.

$\mathrm{O}$ ator/indivíduo, cidadão ou paciente, por sua vez, também pode não ser apenas um agente passivo no processo de adoção ou incorporação de nova(s) tecnologia(s). Em maior ou menor grau, e até por influência cultural, da mídia ou na busca de soluções alternativas pode experimentar, encontrar, ou não, algum significado, ganhar experiência e seguidamente dialogar com seus pares, chegando mesmo, em alguns casos, a influenciar sua coletividade.

Segundo Rogers 2, esta lista diversa de ações e percepções enfatizam a natureza complexa do processo de adoção e demonstra quão inadequadas são, neste nível, as categorias explanatórias early adopters ou laggards (para aqueles que resistissem ou só adotassem tardiamente em relação aos demais), que têm sido erroneamente utilizadas. Embora no nível de sistema de saúde isto pareça ter menor impacto e por isto seria pouco relacionado ao escopo desta revisão, é importante salientar que indivíduos colocam, ou omitem, da pauta de tarefas, segundo suas percepções, ações que podem promover ou dificultar a adoção de inovações. Os sete aspectos dos indivíduos que esse autor salientou como relevantes para o processo de adoção são: (i) seus antecedentes psicológicos gerais (propensão de experimentar o novo, tolerância à ambigüidade e estilo de aprendizado 90 etc.); (ii) antecedentes psicológicos específicos de contexto (motivação ${ }^{91}$, capacidade e competência ${ }^{92}$, como no caso de enfermeiras e outros profissionais de saúde); (iii) o significado atribuído (compatível com sua percepção ${ }^{93}$ ); (iv) o fato de possuir algum nível de influência na decisão de adoção; (v) a disponibilidade de informação prévia 90; (vi) a disponibilidade de informação suficiente e treinamento adequado para as primeiras utilizações 90; e (vii) o retorno (feedback) sobre a(s) conseqüência(s) da adoção e suficiente oportunidade, autonomia e suporte para adaptar e refinar a nova tecnologia recém-estabelecida 90 ao propósito desejado.

Por outro lado, a evolução da tecnologia per si para outros paradigmas de desenvolvimento ${ }^{94}$, tais como a integração ${ }^{95}$, nanotecnologias 96 , miniaturização ou biotecnologias podem representar mudanças e expansão de indicações e mercados consideráveis. Segundo as necessidades, possibilidades e recursos, os países podem expandir seus próprios parques tecnológicos ou se tornarem mais dependentes de importações. As políticas nacionais de desenvolvimento tecnológico são fatores centrais que determinam a facilidade, ou dificuldade, do desenvolvimento próprio ou não, de inovações. A integração governamental com a pesquisa científica tecnológica levou à maior solidificação da indústria no setor saúde no Reino Unido comparativamente à Austrália ${ }^{97}$. Os países produtores de tecnologias retiram maiores benefícios fiscais, com possibilidade de algum retorno financeiro para a assistência e a pesquisa, havendo pressão menor sobre os custos dos serviços de saúde nos sistemas nacionais como o National Health Services (NHS). Com modelo de mercado, como nos Estados Unidos, detentor de $45 \%$ das indústrias do setor saúde, a pressão sobre os custos dos serviços de saúde tem crescido, pois não há interconexões, retorno ou facilidades para o setor saúde, embora se observe a crescente dependência da indústria americana em relação à ciência financiada, basicamente pelo setor público ${ }^{98}$. No Japão, que possui $25 \%$ das indústrias do setor saúde, o desenvolvimento das novas tecnologias e a pesquisa são majoritariamente financiados pela indústria, mas a política nacional de saúde redistribui recursos 99 havendo pressão menor sobre os custos dos serviços de saúde 100 . Observa-se, assim, que as instituições públicas e governamentais têm um peso expressivo em relação às fontes de financiamento para desenvolvimento de inovações e influenciam, portanto, a adoção e a difusão das tecnologias para a saúde. 


\section{Proposta de estrutura para a análise de decisões de incorporação de novas tecnologias}

Considerando-se o microssistema hospitalar, por aproximação, como um sistema (paradigma adotado por Easton 101), é possível conceituar complexos fenômenos políticos a exemplo da aplicação da teoria dos sistemas realizada por este e outros autores 100,102,103,104,105,106. Em segundo lugar, é possível tentar explicar as interrelações entre os atores e abordar o problema proposto de análise das decisões de incorporar novas tecnologias ao considerar o processo decisório como a unidade de análise, segundo a decisão (policy) e as diretivas (politics), como sendo um problema quase-estruturado ocorrendo em um momento e situação determinados por/com os atores que compõem a organização. Problema quase-estruturado é o que não se pode definir nem explicar com precisão, por isto, não se sabe bem como enfrentá-lo e, muito menos, se conhecem os critérios para escolher entre as opções concebidas para enfrentá-lo, conforme a argumentação de Matus 105. A primeira dificuldade com tais problemas está em reconhecê-los.

Este processo de entendimento se refere à classificação de cada processo decisório estudado em uma das cinco concepções analítico-teóricas ou modelos. Essas concepções estudam as relações entre os atores, a informação e a temporalidade durante as etapas dos processos envolvidos na elaboração da decisão, tentando definir diferentes estratégias de resposta e ação para cada situação especificada. Forester 106 sistematizou um esqueleto para a articulação da discussão sobre os diversos elementos, entre as relações percebidas nos discursos dos entrevistados, a qualidade da informação disponível, a temporalidade e estratégias das ações documentadas (Tabela 1).

Em cada etapa do processo decisório, entendida como etapa de formulação, implementação planejada e resultados antecipados, os elementos identificados podem ser descritos sob a luz dos conceitos subjacentes que influenciam a construção de necessidades e a demanda por decisões de incorporação, segundo o nível em que os fatores influenciaram, como proposto por Rogers 2 , na Figura 1.

A coleta do material para a análise pode, assim, constituir-se a partir de um questionário semi-estruturado, que ordena, identifica e qualifica estas variáveis, segundo a tecnologia proposta. Quando se tratar de decisões futuras, o processo de entrevistas aos interessados pode ser elaborado em grupos para consenso. No caso de estudo de decisões retrospectivas, é conveniente entrevistar pessoalmente os participantes da decisão visando a observar dúvidas e adaptar o instrumento, prevenindo-se inconsistências. As ações e percepções dos entrevistados abrangendo o nível II e suas interações com os níveis III e IV deve-

Modelos, elementos e estratégias de processo decisório.

\begin{tabular}{|c|c|c|c|c|c|}
\hline Elementos & $\begin{array}{l}\text { Compreensiva } \\
\text { (sem limites) }\end{array}$ & $\begin{array}{l}\text { Limitação } \\
\text { cognitiva - I }\end{array}$ & $\begin{array}{l}\text { Diferenças } \\
\text { sociais - II }\end{array}$ & Pluralista - III & $\begin{array}{c}\text { Limitação } \\
\text { estrutural - IV }\end{array}$ \\
\hline Ator & $\begin{array}{l}\text { Um ator racional } \\
\text { decide e executa }\end{array}$ & $\begin{array}{l}\text { Um ator não- } \\
\text { racional, falível }\end{array}$ & $\begin{array}{l}\text { Diversos atores e } \\
\text { competências em } \\
\text { cooperação }\end{array}$ & $\begin{array}{l}\text { Atores competindo } \\
\text { organizados em } \\
\text { grupos de interesses }\end{array}$ & $\begin{array}{c}\text { Atores em estruturas } \\
\text { políticas e econômicas } \\
\text { com desigualdade }\end{array}$ \\
\hline Problema & Bem-definido & $\begin{array}{l}\text { Definido de forma } \\
\text { imprecisa }\end{array}$ & $\begin{array}{c}\text { Interpretação variável } \\
\text { a cada ator }\end{array}$ & $\begin{array}{l}\text { Múltiplas definições } \\
\text { de valores, impactos } \\
\text { e direitos }\end{array}$ & $\begin{array}{c}\text { Definições em } \\
\text { bases ideológicas }\end{array}$ \\
\hline Informação & Perfeita & Imperfeita & $\begin{array}{l}\text { Qualidade e acesso } \\
\text { variados }\end{array}$ & $\begin{array}{c}\text { Contestada e aceita } \\
\text { como manipulada }\end{array}$ & $\begin{array}{c}\text { Desinformação ideológica } \\
\text { e vinculada ao poder }\end{array}$ \\
\hline Tempo & Infinito ou sempre suficiente & Limitado & Limitado & Tempo é poder & $\begin{array}{c}\text { É poder e é limitado } \\
\text { conforme interesse } \\
\text { dos atores }\end{array}$ \\
\hline $\begin{array}{l}\text { Estratégia } \\
\text { prática }\end{array}$ & $\begin{array}{l}\text { Tecnicamente } \\
\text { perfeita, ótima }\end{array}$ & $\begin{array}{c}\text { Baseada em baixas } \\
\text { expectativa e satisfação }\end{array}$ & Atuação em redes & $\begin{array}{l}\text { Incremental, com } \\
\text { verificação e ajustes }\end{array}$ & $\begin{array}{l}\text { Baseada em antecipação, } \\
\text { neutralização, organização }\end{array}$ \\
\hline
\end{tabular}


rão ser descritas e discutidas, e seguidas de uma discussão geral sobre o seu possível impacto.

Diversas disciplinas de análise de discurso estão apresentadas na Figura 3, permitindo estruturar de maneira mais sistemática a análise das respostas ao instrumento de entrevista. Visando a elevar a consistência das respostas entre os diversos atores, é conveniente auxiliar o instrumento de entrevista com um glossário e definição dos conceitos utilizados nas perguntas.

Resumidamente, a proposta de apresentação de cada decisão sobre a incorporação de uma nova tecnologia contemplaria:

- O contexto de sua utilização, a indicação, sua importância do ponto de vista epidemiológico e o tratamento atualmente preconizado;

- A nova tecnologia e outros constituintes do programa em que se insere, sua eficácia e segurança;

- Os custos observados e associados ao programa que utilizou a nova tecnologia comparativamente às alternativas estabelecidas;

- Ações e percepções dos atores na decisão de incorporar essa tecnologia, com a análise do discurso dos respondentes situando os elementos iniciais da adoção, atores, linha de relações temporais, organização da prática na implemen- tação e desdobramentos observados, bem como sua discussão segundo as categorias de fatores relevantes elencadas na revisão da literatura; e - A discussão geral sobre o seu possível impacto.

\section{Conclusão}

As decisões de incorporação de tecnologias nos serviços de saúde envolvem múltiplos níveis e stakeholders. Esses atores interessados estão, por sua vez, inter-relacionados de maneira complexa em sistemas criativos com múltiplos determinantes e fatores de confusão. A aplicação e intensidade desses fatores nos processos de decisão de incorporação de produtos e programas nos serviços de saúde conformam a capacidade instalada nas redes locais e regionais e modificam o sistema de saúde. A observação empírica dos processos de decisão de incorporação tecnológica nos serviços de saúde do Brasil constitui um desafio importante a ser abordado. O reconhecimento e dimensionamento destas variáveis podem auxiliar a melhorar o planejamento pró-ativo dos serviços de saúde e do próprio sistema de saúde.

Figura 3

Metáforas, mecanismos e aspectos para a análise de discurso sobre decisões segundo diferentes bases teóricas e conceituais sobre adoção e disseminação de inovações em sistemas.

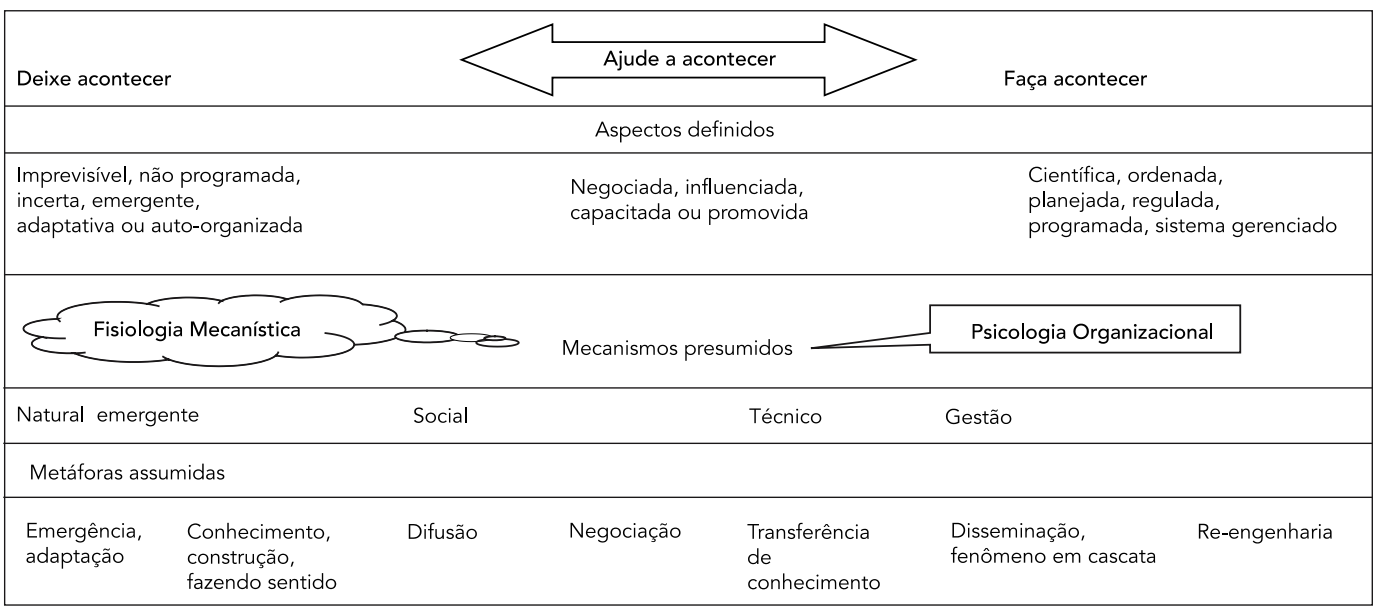

Nota: traduzido e adaptado com permissão de Greenhalgh et al. 107. 


\section{Resumo}

A dinâmica exponencial de incorporação tecnológica na saúde tem sido considerada como uma das razões para o crescimento dos gastos do setor. Estas decisões envolvem múltiplos níveis e stakeholders. A descentralização multiplicou os níveis de decisão, com difíceis escolhas múltiplas e recursos restritos. A inter-relação entre os atores é complexa, em sistemas criativos com múltiplos determinantes e fatores de confusão. Esta revisão discute a interação entre os fatores que influenciam as decisões de incorporação de tecnologias nos serviços de saúde e propõe uma estrutura para sua análise. A aplicação e intensidade desses fatores nos processos de decisão de incorporação de produtos e programas nos serviços de saúde conformam a capacidade instalada nas redes locais e regionais e modifica o sistema de saúde. A observação empírica dos processos de decisão de incorporação tecnológica nos serviços de saúde do Brasil constitui um desafio importante. O reconhecimento estruturado e dimensionamento destas variáveis podem auxiliar a melhorar o planejamento pró-ativo dos serviços de saúde.

Tecnologia Biomédica; Difusão de Inovações; Planejamento em Saúde; Serviços de Saúde

\section{Referências}

1. Silva PF, Waissmann W. Normatização, o Estado e a saúde: questões sobre a formalização do direito sanitário. Ciênc Saúde Coletiva 2005; 10:237-44.

2. Rogers EM. Diffusion of innovations. New York: Free Press; 1962.

3. Greer AL. Advances in the study of diffusion of innovation in health care organizations. Milbank Mem Fund Q 1977; 55:505-32.

4. Greer AL. The state of the art versus the state of the science: the diffusion of new medical technologies into practice. Int J Technol Assess Health Care 1988; 4:5-26.

5. Greer AL. The shape of resistance ... the shapers of change. Jt Comm J Qual Improv 1995; 21:328-32.

6. Kaluzny AD. Innovation in health services: theoretical framework and review of research. Health Serv Res 1974; 9:101-20.

7. Rogers EM, Shoemaker FF. Communications of innovations: a cross-cultural approach. 2nd Ed. New York: Free Press; 1971.

8. Warner KE. The need for some innovative concepts of innovation: an examination of research on the diffusion of innovations. Policy Science 1974; 5:433-51.
9. Fendrick MA, Schwartz JS. Physician's decisions regarding the acquisition of technology. In: Gelijns AC, Dawkins HV, editors. Adopting new medical technology. Washington DC: National Academies Press; 1994:71-84.

10. Gelijns AC, Halm EA, editors. The changing economics of medical technology. Washington DC: National Academies Press; 1991.

11. Greer AL. Adoption of medical technologies: the hospital's three decision systems. Int J Technol Assess Health Care 1985; 4:17-26.

12. Teplensky JD, Pauly MV, Kimberly JR, Hillman AL, Schwartz JS. Hospital adoption of medical technology: an empirical test of alternative models. Health Serv Res 1995; 30:437-65.

13. Greenberg D, Pliskin JS, Peterburg Y. Decision making in acquiring medical technologies in Israeli medical centers: a preliminary study. Int J Technol Assess Health Care. 2003; 19:194-201.

14. Conrad P. Types of medical social control. Sociol Health Illn 1979; 1:1-11.

15. Timmermans S, Berg M. The practice of medical technology. Sociol Health Illn 2003; 25:97-114. 
16. Callon M, Law J. On interests and their transformation: enrolment and counter-enrolment. Soc Stud Sci 1982; 12:612-25.

17. van der Ploeg I. Hermaphrodite patients: in vitro fertilization and the transformation of male infertility. Sci Technol Human Values 1995; 20:460-81.

18. Tellioglu H, Wagner I. Work practices surrounding PACS: the politics of space in hospitals. Comput Support Coop Work 2001; 10:163-88.

19. Blume SS. Insight and industry: on the dynamics of technological change in medicine. Cambridge: MIT Press;1992.

20. Wang SS, Mendelson DN, Schulman KA, Mark DB. Exploring options for improving healthcare. Am Heart J 2004; 147:23-30.

21. Newhouse JP. Medical care costs: how much welfare loss. J Econ Perspect 1992; 6:3-21.

22. Pallas G, Simon F, Sockeel P, Chapuis O, Jancovici R. Inguinal hernia in Africa and laparoscopy: utopia or realism? Med Trop 2000; 60:389-94.

23. Brouselle A. Controlling health expenditures: what matters. In: The National Forum on Health, editor. Striking a balance: health care systems in Canada and elsewhere. v. 4. Sainte-Foy: Editions MultiMondes; 1998. p. 33-84.

24. Menon D, Marshall D. Technology assessment in teaching hospitals. Dimens Health Serv 1990; 67:26-8

25. Trindade E. Perfil Hospitais Sentinelas. http:// www.anvisa.gov.br/divulga/ noticias / 060801 2.htm (acessado em 08/Jan/2008).

26. House R, Rousseau DM, Thomas-Hunt M. The meso paradigm: a framework for the integration of micro and macro organisational behaviour. Research in Organizational Behaviour 1995; 17: 71-114.

27. Champagne F, Denis J-L, Pineault R, Contandriopoulos A. Structural and political models of analysis of the introduction of an innovation in organizations: the case of the change in the method of payment of physicians in long-term care hospitals. Health Serv Manage Res 1991; 4:94-111.

28. Barnsley J, Lemieux-Charles L, McKinney MM. Integrating learning into integrated delivery systems. Health Care Manage Rev 1998; 23:18-28.

29. Zahra AS, George G. Absorptive capacity: a review, reconceptualization and extension. Acad Manage Rev 2002; 27:185-203.

30. Dopson S, Fitzgerald L, Ferlie E, Gabbay J, Locock L. No magic targets. changing clinical practice to become more evidence based. Health Care Manage Rev 2002; 37:35-47.

31. Pettigrew AM, McKee L. Shaping strategic change. making change in large organisations. London: Sage Publications; 1992.

32. Gosling AS, Westbrook JI, Braithwaite J. Clinical team functioning and IT innovation: a study of the diffusion of a point-of-care online evidence system. J Am Med Inform Assoc 2003; 10:244-51.

33. Nystrom PC, Ramamurthy K, Wilson AL. Organizational context, climate and innovativeness: adoption of imaging technology. Journal of Engineering and Technology Management 2002; 19:221-47.

34. van de Ven AH, Polley DE, Garud R, Venkataraman S. The innovation journey. Oxford: Oxford University Press; 1999.
35. Gustafson DH, Sainfort F, Eichler M, Adams L, Bisognano M, Steudel H. Developing and testing a model to predict outcomes of organizational change. Health Serv Res 2003; 38:751-76.

36. Arrow KJ. Problems of resource allocation in United States medical care. Experientia Suppl 1972; 17:392-410.

37. Oxley H, MacFarland M. Health care reform controlling spending and increasing efficiency. Paris: Organisation for Economic Co-operation and Development; 1994.

38. McLaughlin CP, Penchansky R. Diffusion of innovation in medicine: a problem of continuing medical education. J Med Educ 1965; 40:437-47.

39. Moore GA. Crossing the chasm. New York: Harper Business; 1991.

40. Becker MH. Factors affecting the diffusion of innovation among health professionals. Am J Public Health 1970; 60:294-304.

41. Silverberg G. Adoption and diffusion of technology as a collective evolutionary process. In: Freeman C, Soete L, editors. New explorations in the economics of technological change. London: Pinter Publishers; 1990. p. 177-92.

42. Rogers E. Diffusion of innovations. $4^{\text {th }}$ Ed. New York: Free Press; 1995.

43. Dirksen CD, Ament AJ, Go PM. Diffusion of six surgical endoscopic procedures in the Netherlands. Health Policy 1996; 37:91-104.

44. Meyer M, Johnson D, Etthington C. Contrasting attributes of preventive health innovations. J Commun 1997; 47:112-31.

45. Grimshaw JM, Thomas RE, MacLennan J, Fraser C, Ramsay CR, Vale I, et al. Effectiveness and efficiency of guideline dissemination and implementation strategies. Health Technol Assess 2004; 8:1-72.

46. Fennel ML, Warnecke RB. The diffusion of medical innovations: an applied network analysis. New York: Plenum; 1988.

47. Foy R, MacLennan G, Grimshaw J, Penney G, Campbell M, Grol R. Attributes of clinical recommendations that influence change in practice following audit and feedback. J Clin Epidemiol 2002; 55:717-22.

48. Ferlie E, Gabbay J, Fitzgerald L, Locock L, Dopson S. Evidence-based medicine and organisational change: an overview of some recent qualitative research. In: Ashburner L, editor. Organisational behaviour and organisational studies in health care: reflections on the future. Basingstoke: Palgrave; 2001. p. 18-42.

49. Aubert BA, Hamel G. Adoption of smart cards in the medical sector: the Canadian experience. Soc Sci Med 2001; 53:879-94.

50. Denis JL, Hebert Y, Langley A, Lozeau D, Trottier LH. Explaining diffusion patterns for complex health care innovations. Health Care Manage Rev 2002; 27:60-73.

51. Grilli R, Lomas J. Evaluating the message: the relationship between compliance rate and the subject of a practice guideline. Med Care 1994; 32:202-13.

52. Meyer AD, Goes JB. organisational assimilation of innovations: a multi-level contextual analysis. Acad Manage Rev 1988; 31:897-923. 
53. Rogers EM. A prospective and retrospective look at the diffusion model. J Health Commun 2004; 9 Suppl 1:13-9.

54. Watts HG. The transfer of appropriate orthopaedic technology to less developed countries a philosophy of involvement. Instr Course Lect 2000; 49:579-83.

55. Plsek PE, Wilson T. Complexity science: complexity, leadership, and management in healthcare organizations. BMJ 2001; 323:746-9.

56. Yetton P, Sharma R, Southon G. Successful IS innovation: the contingent contributions of innovation characteristics and implementation process. Journal of Information Technology 1999; 14:53-68.

57. Øvretveit J, Bate P, Cleary P, Cretin S, Gustafson D, McInnes K, et al. Quality collaboratives: lessons from research. Qual Saf Health Care 2002; 11: 345-51.

58. Dearing JW, And O. Portraying the new: communication between university innovators and potential users. Sci Commun 1994; 16:11-42.

59. Arthur WB. Competing technologies, increasing returns, and lock-in by historical events. Economic Journal 1989; 99:116-31.

60. James AE, Perry S, Warner SE, Chapman JE, Zaner RM. The diffusion of medical technology: free enterprise and regulatory models in the USA. J Med Ethics 1991; 17:150-5.

61. Robinson MA. Issues and strategies for faculty development in technology and biomedical informatics. Adv Dent Res 2003; 17:34-7.

62. May C, Ellis NT. When protocols fail: technical evaluation, biomedical knowledge, and the social production of "facts" about a telemedicine clinic. Soc Sci Med 2001; 53:989-1002.

63. Valente TW. Social network thresholds in the diffusion of innovations. Soc Networks 1996; 18:69-89.

64. West E, Barron DN, Dowsett J, Newton JN. Hierarchies and cliques in the social networks of health care professionals: implications for the design of dissemination strategies. Soc Sci Med 1999; 48:633-46.

65. Fitzgerald L, Ferlie E, Wood M, Hawkins C. Interlocking interactions, the diffusion of innovations in health care. Hum Relat 2002; 55:1429-49.

66. Becker C. If you have it, you use it. Latest Blues association study finds new technology not only drives demand but also increases spending. Mod Healthc 2003; 33:9-11.

67. Coleman JS, Katz E, Menzel H. Medical innovations: a diffusion Study. New York: Bobbs-Merrill; 1966.

68. Locock L, Dopson S, Chambers D, Gabbay J. Understanding the role of opinion leaders in improving clinical effectiveness. Soc Sci Med 2001; 53:74557.

69. Doumit G, Gattellari M, Grimshaw J, O’Brien MA. Local opinion leaders: effects on professional practice and health care outcomes. In: Cochrane Database of Systematic Reviews 1997; Issue 3.

70. Backer TE, Rogers EM. Diffusion of innovations theory and work-site AIDS programs. J Health Commun 1998; 3:17-28.

71. Markham SK. A longitudinal examination of how champions influence others to support their projects. Journal of Product Innovation Management 1998; 15:490-504.
72. Schon DA. Champions for radical new inventions. Harv Bus Rev 1963; 41:77-86.

73. Shane S. Uncertainty avoidance and the preference for innovation championing roles. Journal of International Business Studies 1995; 26:47-68.

74. Kimberly JR, Evanisko JM. Organisational innovation: the influence of individual, organisational and contextual factors on hospital adoption of technological and administrative innovation. Acad Manage J 1981; 24:689-713.

75. Bashshur RL. Critical issues in telemedicine. Telemed J 1997; 3:113-26.

76. Pedersen T, Møller AM, Cracknell J. The mission of the Cochrane Anesthesia Review Group: preparing and disseminating systematic reviews of the effect of health care in anesthesiology. Anesth Analg 2002; 95:1012-8.

77. Hailey D, Menon D. A short history of INAHTA. International Network of Agencies for Health Technology Assessment. Int J Technol Assess Health Care 1999; 15:236-42.

78. Rydén L, Stokoe G, Breithardt G, Lindemans F, Potgieter A; Task Force 2 of the Cardiovascular Round Table of the European Society of Cardiology. Patient access to medical technology across Europe. Eur Heart J 2004; 25:611-6.

79. Proceedings of the 26th Annual ISCE Conference: Research and Technology Transfer in Computerized Electrocardiology. J Electrocardiol 2001; 34 Suppl:1-269.

80. Giddens A. As conseqüências da modernidade. São Paulo: Editora da Unesp; 1991.

81. Perspectives. Americans love medical innovation... Med Health 2001; 55:7-8.

82. Ferguson JH, Dubinsky M, Kirsch PJ. Court-ordered reimbursement for unproven medical technology: circumventing technology assessment. JAMA 1993; 269:2116-21.

83. Coile Jr. RC. Technowave 2010: hospitals compete on high-tech care. Mich Health Hosp 2003; 39:12-5.

84. Cosgrove DM. The innovation imperative. J Thorac Cardiovasc Surg 2000; 120:839-42.

85. Hofmann B. Is there a technological imperative in health care? Int J Technol Assess Health Care 2002; 18:675-89.

86. Muraskas J, Marshall PA, Tomich P, Myers TF Gianopoulos JG, Thomasma DC. Neonatal viability in the 1990s: held hostage by technology. Camb Q Healthc Ethics 1999; 8:160-70.

87. Weller RE. Changing the health care equation through technological innovation. P R Health Sci J 1995; 14:131-6.

88. Goodman CS, Gelijns AC. The changing environment for technological innovation in health care. Baxter Health Policy Rev 1996; 2:267-315.

89. Hagland M. Up \& comers. Whether with newer tools or familiar technology with a new twist, companies have a solution for everything. Healthc Inform 2004; 21:67-71.

90. Hall GE, Hord SM. Change in schools. Albany: State University of New York Press; 1987.

91. Gladwin J, Dixon RA, Wilson TD. Rejection of an innovation: health information management training materials in East Africa. Health Policy Plan 2002; 17:354-61. 
92. Wejnert B. Integrating models of diffusion of innovations: a conceptual framework. Annu Rev Sociol 2002; 28:297-326.

93. Eveland JD. Diffusion, technology transfer, and implementation: thinking and talking about change. Knowledge 1986; 8:303-22.

94. NBIC Convergence 2003: converging technologies for improving human performance. Ann N Y Acad Sci 2004; 1013:1-259.

95. Roco MC. Science and technology integration for increased human potential and societal outcomes. Ann N Y Acad Sci 2004; 1013:1-16.

96. Lymberis A, Olsson S. Intelligent biomedical clothing for personal health and disease management: state of the art and future vision. Telemed J E Health 2003; 9:379-86.

97. Ogilvie DB. Requirements for a biotechnology future: the current position of Australia and the United Kingdom. Aust N Z J Med 2000; 30:718-23.

98. Narin F, Hamilton KS, Olivastro D. The increasing linkage between U.S. technology and public science. Research Policy 1997; 26:317-30.

99. Ikegami N, Campbell JC. Health care reform in Japan: the virtues of muddling through. Health Aff 1999; 18:56-75.
100. Ikegami N, Drummond M, Fukuhara S, Nishimura S, Torrance GW, Schubert F. Why has the use of health economic evaluation in Japan lagged behind that in other developed countries? Pharmacoeconomics 2002; 20 Suppl 2:1-7.

101. Easton D. The political system. New York: Wiley; 1953.

102. Dagnino R. Metodologia de análise de políticas públicas. Campinas: Grupo de Análise de Políticas de Inovação, Universidade Estadual de Campinas; 2002.

103. Ham C, Hill M. The policy process in the modern capitalist state. London: Sage Publications; 1993.

104. Rhydderch M, Elwyn G, Marshall M, Grol R. Organisational change theory and the use of indicators in general practice. Qual Saf Health Care 2004;13:213-7.

105. Matus C. Política, planejamento e governo. 2a Ed. Brasília: Instituto de Pesquisa Econômica Aplicada; 1996.

106. Forester J. Planning in the face of power. Los Angeles: University of California Press; 1989.

107. Greenhalgh T, Robert G, MacFarlane F, Bate P, Kiriakidou O. Innovations in service organizations. Milbank Q 2004; 82:581-62.

Recebido em 14/Nov/2006

Versão final reapresentada em 19/Set/2007

Aprovado em 08/Nov/2007 